\title{
Osteología del Miembro Torácico de la Corzuela Parda Mazama gouazoubira (G. Fischer, 1814) (Cetartiodactyla: Cervidae)
}

\author{
Osteology of the Thoracic Limb of South American Gray Brocket \\ Mazama gouazoubira (Fischer, 1814) (Cetartiodactyla: Cervidae)
}

\begin{abstract}
Paulo Souza Junior ${ }^{1}$; Mayra Galhardo Ferreira Ribeiro'; Liliana Jost Dutra ${ }^{1}$; Karine de Mattos ${ }^{1}$; Natan da Cruz de Carvalho $^{1}$; Marcelo Abidu-Figueiredo ${ }^{2}$ \& André Luiz Quagliatto Santos ${ }^{3}$
\end{abstract}

SOUZA JUNIOR, P.; RIBEIRO, M. G. F.; DUTRA, L. J.; MATTOS, K.; CARVALHO, N. C.; ABIDU-FIGUEIREDO, M. \& SANTOS, A. L. Q. Osteología del miembro torácico de la corzuela parda Mazama gouazoubira (G. Fischer, 1814) (Cetartiodactyla: Cervidae). Int. J. Morphol., 35(3):1000-1009, 2017.

RESUMEN: El objetivo del estudio fue describir las características anatómicas y establecer hipótesis morfo-funcionales del esqueleto del miembro torácico de Mazama gouazoubira. Para ello, se utilizaron los miembros torácicos de cuatro adultos jóvenes. Los huesos se prepararon mediante ebullición y fueron blanqueados en una solución de peróxido de hidrógeno para obtener descripciones comparativas, mediciones osteométricas y radiografías digitales. En comparación con los rumiantes domésticos, la escápula resultó ser ancha y plana, con el acromion pequeño, el proceso coracoides muy pequeño y sin tuberosidad de la espina. El húmero se observó alargado con la diáfisis redondeada en una sección transversal y con proyecciones no articulares lisas. La ulna se unió al radio hasta la epífisis distal y juntos formaron un espacio interóseo proximal alargado y estrecho, sin surco vascular. El esqueleto de la mano presentó los metacarpianos III y IV bien desarrollados y fusionados, mientras que los metacarpianos II y V resultaron rudimentarios con disposición telemetacarpiana, filogenéticamente típico de cérvidos del Nuevo Mundo. Se encontraron cuatro dedos con tres falanges en cada uno, de los cuales, dos corresponden a los principales (III y IV) que llegan al suelo y dos son rudimentarios (II y V). Las radiografías nos permitieron visualizar los patrones de tensiones trabeculares normales y la osteometría permitió establecer relaciones con fines comparativos. Se reconocieron las adaptaciones esqueléticas del miembro torácico para favorecer la locomoción cursorial saltatoria.

PALABRAS CLAVE: Anatomía animal; Animales silvestres; Cérvidos; Osteometría; Telemetacarpianos.

\section{INTRODUCCIÓN}

Mazama gouazoubira (G. Fischer, 1814) es un rumiante silvestre del orden Cetartiodactyla de la familia Cervidae. Conocido como corzuela parda, corzuela común, cabra silvestre, guazú y guazú virá. Tiene una masa corporal entre 17 y $23 \mathrm{~kg}$, pequeños cuernos no ramificados, pelaje marrón con el tronco grisáceo y la parte ventral de la cola blanca. Se encuentra ampliamente distribuido desde el sur de Uruguay hasta el norte de Mato Grosso en Brasil y ocupa áreas forestales como campos, sabanas y catingas (Tiepolo \& Tomas, 2006; Ferreira et al., 2011). Su población no se considera en peligro de extinción (Black-Decima \& Vogliotti, 2016), excepto en Rio Grande do Sul y Rio de Janeiro, por la caza ilegal, la agricultura y la urbanización de su hábitat (Tiepolo \& Tomas; Teixeira et al., 2010). Su baja tasa reproductiva limitada a una $\mathrm{o}$, esporádicamente, dos crías por año supone una amenaza aún mayor (Mähler Junior \& Schneider, 2003).
Los cérvidos son cursoriales, pero con velocidad y movimientos saltatorios más pronunciados que los rumiantes domésticos. Durante la locomoción de los ungulados, los miembros torácicos son reconocidos por producir fuerzas desaceleradoras, además de resistir al impacto de la locomoción cursorial, mientras que los miembros pelvianos aceleran el desplazamiento (Cavagna et al., 1977). Los estudios morfo-funcionales del miembro torácico pueden contribuir a la comprensión de los hábitos de vida de las especies, siendo importante para el desarrollo de estrategias de conservación. La descripción anatómica macroscópica y el aspecto radiográfico normal ayudan al diagnóstico y planificación de los procedimientos clínicos y quirúrgicos de los veterinarios en áreas protegidas o parques zoológicos. Los datos osteométricos, en general, resultan de utilidad en estudios filogenéticos y alométricos comparativos (von den Driesch, 1976). 
En la revisión bibliográfica, se encontraron muy pocos trabajos sobre la anatomía de Mazama gouzoubira. En ellos se abordan la morfología hepática (Borges et al., 2002), la testicular (Costa, 2009), la topografía vertebro-medular (Lima et al., 2010), las membranas fetales y la placenta (Ferreira et al.), los componentes del cordón espermático (Borelli \& Gonçalves, 2011), la formación de la vena yugular externa (Guimarães et al., 2012), la estructura de las papilas linguales (Kokubun et al., 2012), el origen y la distribución del nervio isquiático (Martins et al., 2013), la morfología del aparato ungueal (Rezende et al., 2013) y la organización del plexo braquial (Vieira et al., 2013). Sin embargo, no se encontraron publicaciones sobre la osteología del miembro torácico de la corzuela parda.

En este contexto, el objetivo ha sido describir las características anatómicas del miembro torácico de Mazama gouazoubira, para establecer hipótesis morfo-funcionales y filogenéticas comparativas.

\section{MATERIAL Y MÉTODO}

Este estudio se realizó en ocho miembros torácicos de cuatro especímenes adultos jóvenes, dos de cada sexo, de la especie Mazama gouazoubira, encontrados muertos en las carreteras de los municipios de Uruguaiana-RS, Dom Pedrito-RS y Uberlândia-MG (autorización SISBIO 336671 y acuerdo de cooperación UFU/IBAMA 002/2011). Posteriormente, los cadáveres fueron transportados al laboratorio de anatomía animal de la Universidad Federal de Pampa para el proceso de maceración ósea en agua hirviendo. A continuación, los restos de tejidos blandos fueron raspados y los huesos sometidos a blanqueamiento en solución de peróxido de hidrógeno $\left(\mathrm{H}_{2} \mathrm{O}_{2}\right)$ a 130 vol. $\left(\mathrm{Brquim}^{\circledR}\right)$ diluido a 1:20, durante 30 minutos. Una vez limpios, se describieron los huesos y se compararon con los rumiantes domésticos disponibles en la colección del laboratorio y la descripción de Varela (2010) para Ozotoceros bezoarticus. La nomenclatura de las descripciones sigue las normas de la International Commitee on Veterinary Gross Anatomy Nomenclature (ICVGAN, 2012). Las mediciones osteométricas se realizaron con un calibrador digital de precisión (resolución 0,01 $\mathrm{mm}$, exactitud $\pm 0,02 \mathrm{~mm}$, ZAAS Precision, Amatools ${ }^{\circledR}$ ) de acuerdo con la norma internacionalmente aceptada definida por von den Driesch (Tabla I).

De los valores de las mediciones se calculó la media aritmética para cada medida realizada en los ocho miembros y fue representada en fotografías de huesos obtenidas mediante cámara digital (resolución 14.2 Mp, modelo ST64, Samsung $\left.{ }^{\circledR}\right)$.
Finalmente, las radiografías óseas, todas ellas realizadas en al menos dos proyecciones (lateral-medial y cráneo-caudal y dorsal-palmar), se obtuvieron con el dispositivo de la marca Phillips ${ }^{\circledR}$, modelo Aquilla Plus 300, con pantallas de $40 \mathrm{KV}$, rayos x $200 \mathrm{~mA}$ durante $0,1 \mathrm{~s}$ en sistema de cinta computarizada marca Kodak ${ }^{\circledast}$ Direct View y guardadas en formato DICOM. La visualización de las imágenes radiográficas y su conversión a formato JPEG se realizó con el software Radiant Dicom Viewer® versión 1.6.8.

\section{RESULTADOS Y DISCUSIÓN}

El miembro torácico de M. gouazoubira se compone de cuatro segmentos: cíngulo escapular, brazo, antebrazo y mano.

El cíngulo escapular (zonoesqueleto) presenta como base ósea una escápula ancha y plana, con acromion pequeño y proceso coracoides muy pequeño. Este segmento difiere de los rumiantes domésticos (Nickel et al., 1986) y de Ozotoceros bezoarticus (Varela), aparentemente sin tuberosidad en la espina de la escápula (Fig. 1). El índice escapular (relación media entre la longitud y la anchura) es de 1:0,6, idéntica a la descrita por Sisson (1986) para el ganado. La espina se dirige en su tercio medio en sentido caudal, como en $O$. bezoarticus (Varela), en bovinos y ovinos, pero de manera diferente a lo que sucede en caprinos (Nickel et al.). Su forma se asemeja a la de un triángulo isósceles, que es el patrón estándar reconocido en otros rumiantes por Searfoss (1995). No se encontró clavícula ósea verdadera, dejando sólo una banda de fibras entre las porciones del músculo braquiocefálico, al igual que en las especies de ungulados (Nickel et al.). Esto puede ser interpretado como una adaptación para aumentar la amplitud de los pasos deseables para las corzuelas, ya que la clavícula limita los movimientos de la escápula (Liem et al., 2013). La fosa subescapular fue comparativamente mayor que en los rumiantes domésticos, asemejándose a $O$. bezoarticus (Varela). Antes de la ebullición, el cartílago escapular resultó ser discreto, semilunar y fijado al margen dorsal.

El húmero (Fig. 2) constituyó el relleno óseo de la región braquial (estilopodio). Al igual que en $O$. bezoarticus (Varela), mostrándose más alargado que en los pequeños rumiantes domésticos y con la diáfisis redondeada en una sección transversal, lo que se diferencia de bovinos y se asemeja a pequeños rumiantes (Nickel et al.). La porción craneal del tubérculo mayor es puntiaguda como en los bovinos y la porción caudal subdesarrollada. No se encontró tubérculo intermedio, aunque Nickel et al., mencionan su formación en rumiantes. El área de inserción del músculo 
SOUZA JUNIOR, P .; RIBEIRO, M. G. F.; DUTRA, L. J.; MATTOS, K.; CAR VALHO, N. C.; ABIDU-FIGUEIREDO, M. \& SANT OS, A. L. Q. Osteología del miembro torácico de la corzuela parda Mazama gouazoubira (G. Fischer, 1814) (Cetartiodactyla: Cervidae). Int. J. Morphol., 35(3):1000-1009, 2017.

infraespinoso es ovalada como en los pequeños rumiantes (Nickel et al.). En general, las proyecciones no articulares como la línea tricipital, tuberosidad deltoidea y cresta del húmero, demostraron ser comparativamente más lisas. La tuberosidad redonda mayor, por ejemplo, se eleva tan poco que parece más una discreta fosa rugosa. Posiblemente el húmero más alargado contribuya a los pasos más largos y las protuberancias más discretas reflejen una menor fuerza ejercida por los músculos sobre estas superficies, indicando una función más de soporte al impacto que para movimientos vigorosos. El agujero nutricio se encuentra en el margen caudal de la diáfisis, en el tercio medio del antímero izquierdo y en el tercio distal del derecho, siendo ésta última la ubicación habitual en los bovinos (Sisson). La superficie articular distal se asemejó a la de los ovinos (Nickel et al.).
La región antebraquial presentó la ulna y el radio como base ósea (zeugopodio) (Fig. 3). La parte proximal del espacio interóseo fue más alargada y estrecha que en los rumiantes domésticos y similares a $O$. bezoarticus (Varela). No se encontró el surco vascular, reconocido en bovinos, proyectándose entre las partes proximal y distal del espacio interóseo. La tuberosidad radial es poco profunda. Las protuberancias craneales y caudales de la tuberosidad del olécranon resultaron muy diferentes, la craneal más aguda y proximalmente dispuesta que en rumiantes domésticos. La ulna resultó ser delgado distalmente y firmemente adherido al radio. El grosor delgado de la ulna debido a la reducción de los elementos distales característicos de los velocistas ungulados (Liem et al.), asociado a su fuerte conexión con el radio y combinado con un espacio interóseo discreto, prácticamente

Tabla I. Medidas adoptadas para el registro osteométrico del miembro torácico del Mazama gouazoubira $(\mathrm{n}=2)$.

\section{Escápula}

HS: altura a lo largo de la espina de la escápula; Ld: mayor longitud dorsal; DHA: altura diagonal (desde el punto más ventral de la escápula hasta el ángulo caudal); SLC: longitud más pequeña a nivel del cuello de la es cápula; GLP: mayor longitud de la región ventral (del tubérculo supraglenoideo hasta el margen de la cavidad glenoidea caudal); BG: mayor anchura de la cavidad glenoidea; LG: longitud de la cavidad glenoidea.

\section{Húmero}

GL: mayor altura; GLC: mayor altura desde la cabeza humeral; Bp: mayor anchura de la extremidad proximal; Dp: espesor de la extremidad proximal; SD: menor diámetro de la diáfisis; Bd: anchura de la extremidad distal; BT: mayor anchura de la superficie articular.

\section{Radio}

GL: mayor longitud; SD: menor anchura de la diáfisis; CD: menor circunferencia de la diáfisis; Bd: mayor anchura de la extremidad distal; BFd: mayor anchura de la cara articular distal.

\section{Ulna}

GL: mayor longitud; LO: longitud del olécranon; DPA: profundidad desde el proceso anconeo; SDO: menor profundidad del olécranon; BPC: anchura entre los procesos coronoides.

\section{Huesos Carpales}

GBca: mayor anchura del accesorio del carpo; GBcr: mayor anchura del carpo radial; GBci: mayor anchura del carpo intermedio; GBcu: mayor anchura del carpo cubital; GBII/III: mayor anchura del carpiano II e III fusionados; GBIV: mayor anchura del carpiano IV; Lca: mayor longitud del accesorio del carpo; Lcr: m ayor longitud del carpo radial; Lci: mayor longitud del carpo intermedio; Lcu: mayor longitud del carpo cubital; LII/III: mayor longitud del carpiano II e III fusionados; LIV: mayor longitud del carpiano IV.

\section{Huesos Metacarpianos y Falanges proximales y medias}

GL: mayor longitud; SD: menor diámetro de la diáfisis; Bp: mayor anchura de la extremidad proximal; Bd: mayor anchura de la extremidad distal; GLI: mayor longitud de la parte lateral; Ll: mayor longitud de la parte medial; DD: menor profundidad de la diáfisis

Falanges distales

Ld: Longitud de la superficie dorsal; DLS: mayor longitud diagonal de la sola; MBS: anchura al nivel de la mitad de la sola. 


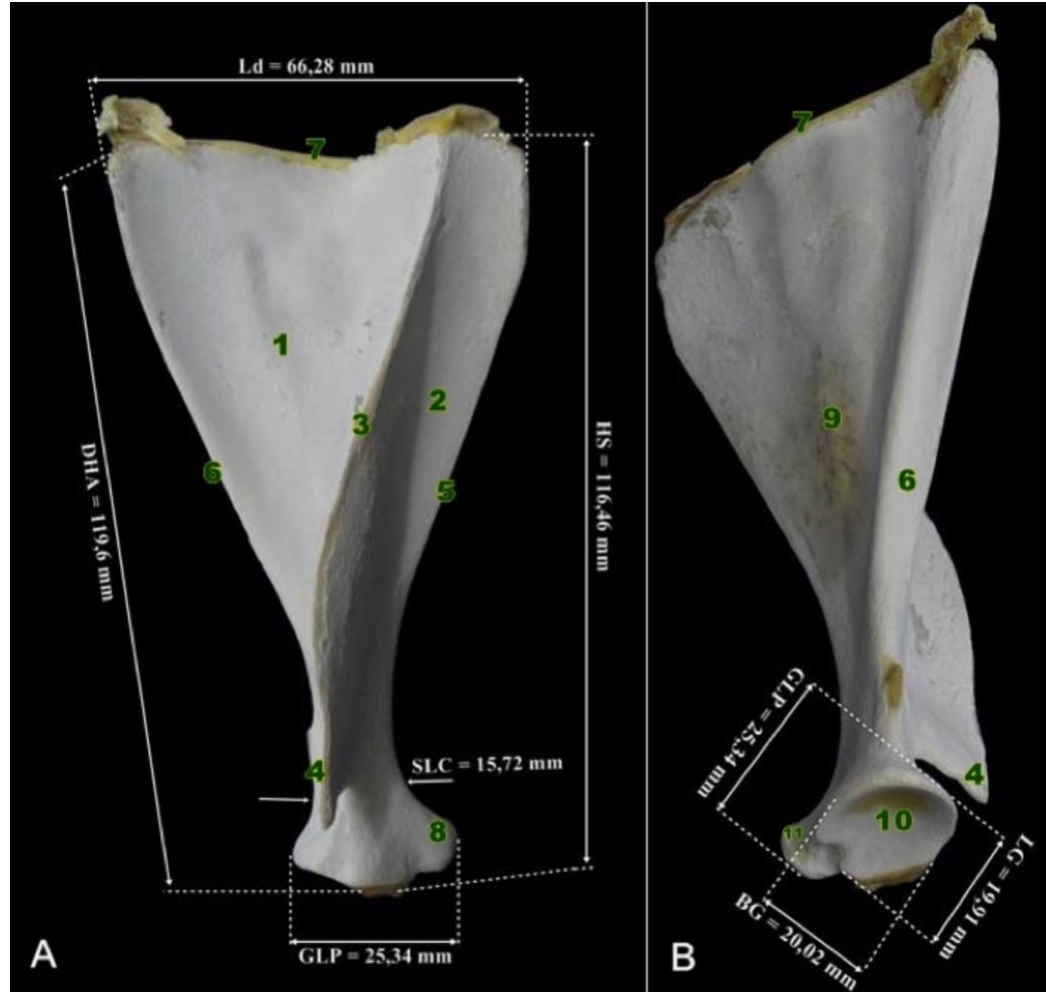

Fig. 1. La escápula derecha de un espécimen hembra de Mazama gouazoubira en vista lateral (A) y caudomedial (B) que muestra las medidas de acuerdo con von den Driesch (1976) y los accidentes anatómicos: fosa infraespinosa (1) fosa supraespinosa (2), espina de la escápula (3), acromion (4), borde craneal (5), borde caudal (6), borde dorsal (7), tubérculo supraglenoideo (8), fosa subescapular (9) cavidad glenoidea (10) y proceso coracoides (11).

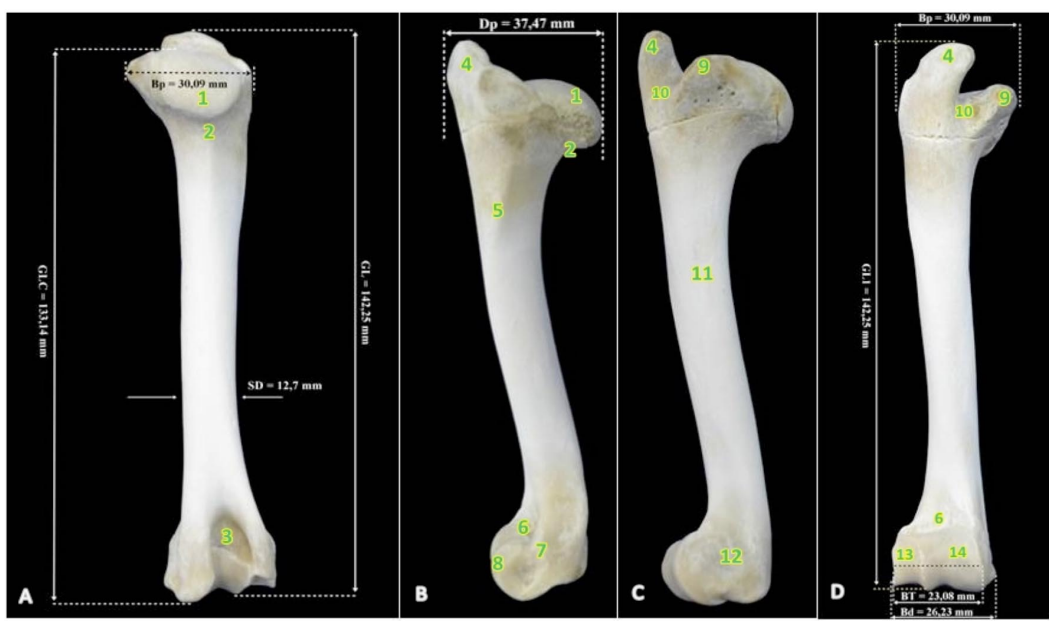

Fig. 2. Húmero de un espécimen hembra de Mazama gouazoubira en vista caudal (A) y lateral (B) del antímero izquierdo medial (C) y craneal (D) del lado derecho, revelando las medidas de acuerdo con von den Driesch (1976) y los accidentes anatómicos: cabeza del húmero (1), cuello del húmero (2), fosa del olécranon (3), tuberosidad mayor (4), tuberosidad deltoidea (5), fosa radial (6), epicóndilo lateral ( 7), capítulum (8), tuberosidad menor (9), surco intertubercular (10), tuberosidad redonda mayor (11), epicóndilo medial (12), cóndilo lateral / capítulum (13) y cóndilo medial / tróclea (14). anulan los movimientos de rotación. Lo que es deseable en un miembro especializado para priorizar únicamente los arcos de flexión y extensión en un plano paramediano.

La mano (autopodio) se formó por los huesos del carpo, metacarpo y falanges. El carpo (basipodio) fue similar en los rumiantes domésticos (Sisson) y en $O$. bezoarticus (Varela), constituido por una hilera proximal que contiene los carpianos radiales, intermedio, cubital y accesorio, y otra distal con el segundo y tercer carpiano fusionados y el cuarto carpiano distinto (Fig. 4). La ausencia del primer hueso carpiano y la fusión del segundo con el tercero se explican funcionalmente, ya que cuanto mayor es la reducción del número de huesos carpianos, menor es la libertad de movimiento de la muñeca respecto a la supinación y pronación (Liem et al.). Tales movimientos rotacionales no existen en los rumiantes (Liebich et al., 2011).

Se encontraron cuatro metacar-pianos, siendo el tercero y el cuarto los principales y fusionados, formando un hueso principal, típico de los rumiantes. El segundo y quinto metacarpianos son extremadamente delgados y cortos, habiendo perdido sus porciones proximales, aumentando progresivamente de grosor al acercarse a la epífisis distal (Fig. 5), que se articula con la falange proximal. Por lo tanto, se posicionan axialmente únicamente después del tercio medio de los metacarpianos principales. Este es un factor importante, debido a que la principal clasificación dentro de la familia Cervidae se basa en la morfología de los metacarpianos accesorios (Brooke, 1878). En los cérvidos telemetacarpianos la porción distal de los metacarpianos rudimentarios permanece, mientras que en los plesiometacarpianos la porción proximal de estos huesos es la evidente. Estas agrupaciones filogenéticas basadas en la morfología han sido corroboradas por estudios moleculares recientes (Gilbert et al., 2006) y de comportamiento (Cap et al., 2002). De acuerdo con Geist (1998), los cérvidos del Nuevo Mundo son telemetacarpianos y los del Viejo Mundo plesiometacarpianos. Las observaciones en los especímenes de Mazama 


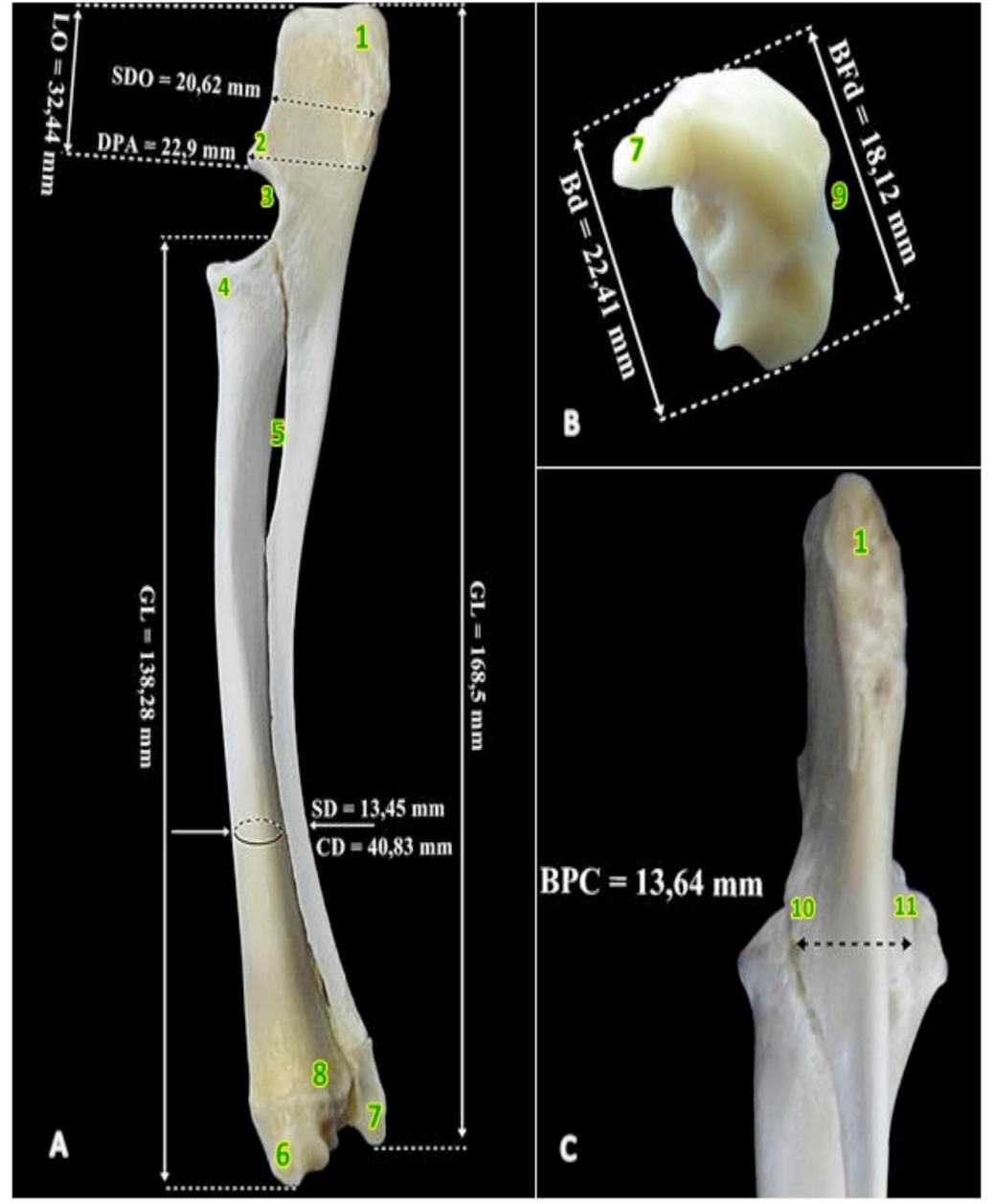

Fig. 3. El radio y la ulna derechos de un espécimen hembra de Mazama gouazoubira en vista medial (A), distal de la superficie articular para el carpo (B) y caudal de la región proximal (C) que muestran las medidas de acuerdo con von den Driesch (1976) y los accidentes anatómicos: tuberosidad del olécranon (1), proceso ancóneo (2), incisura troclear (3), cabeza del radio (4), espacio interóseo (5), proceso estiloides medial (6), proceso estiloides lateral (7), cresta transversa (8), surcos extensores (9), proceso coronoides medial (10) proceso coronoides lateral (11).
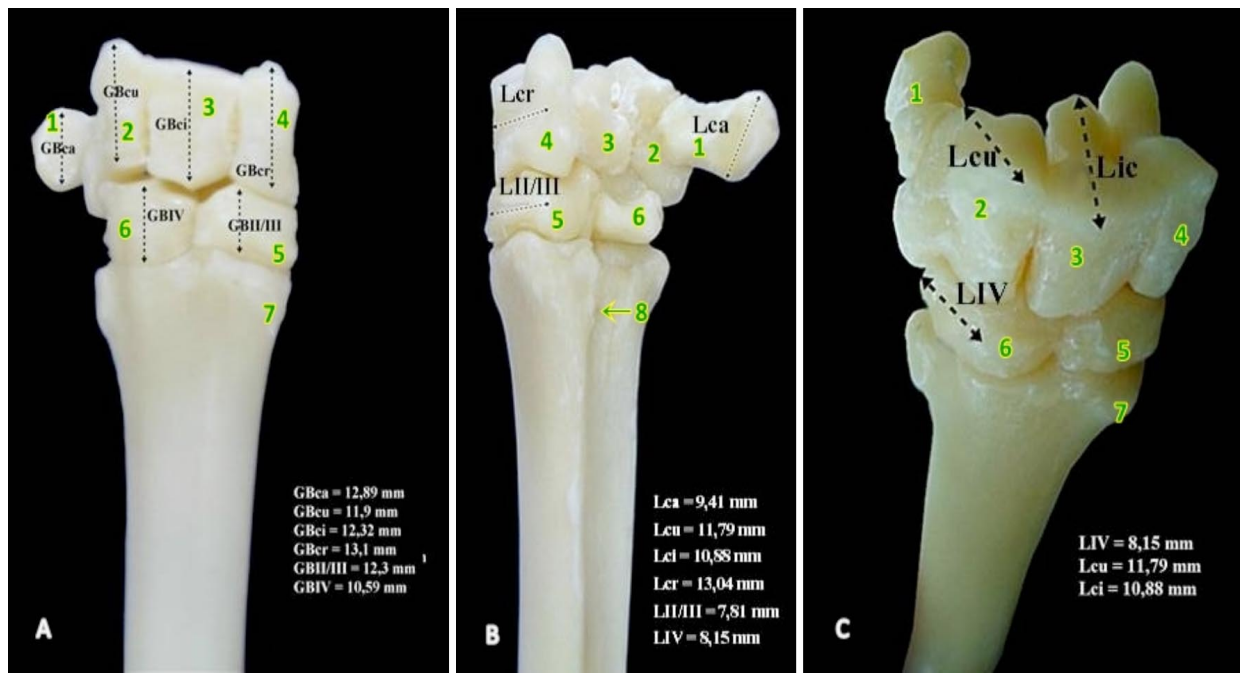

Fig. 4. Los huesos proximales de la mano derecha (carpianos y metacarpianos III y IV), de un espécimen hembra Mazama gouazoubira en vista dorsal (A), palmaro-medial (B) y dorso-lateral (C) que muestra las medidas de acuerdo con von den Driesch (1976) en los huesos accesorio del carpo (1), carpo-cubital (2), carpal intermedio (3), carpo radial (4), carpianos II y III fusionados (5), carpiano IV (6) y los accidentes anatómicos de la tuberosidad metacarpiana (7) y el agujero metacarpiano proximal (8). 


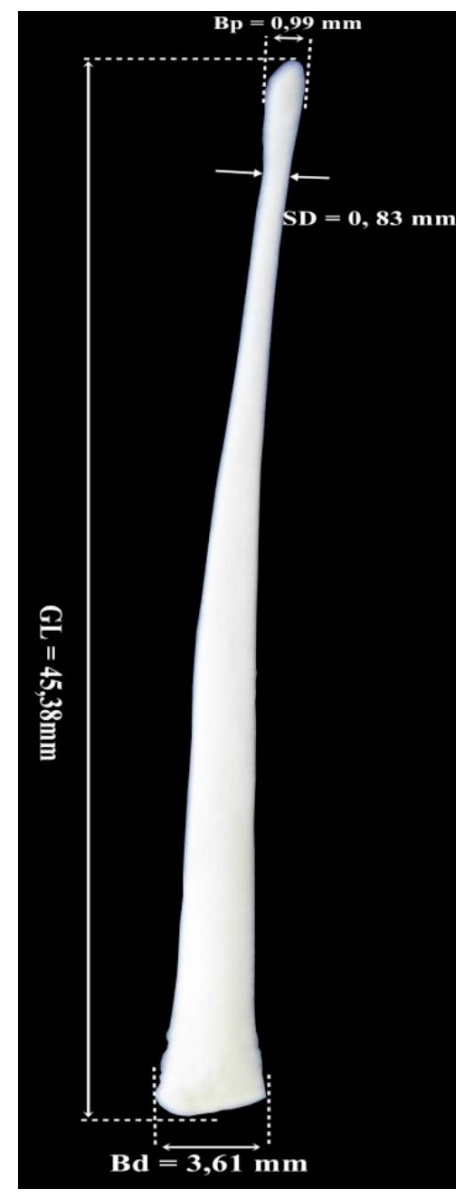

Fig. 5. El metacarpiano II (rudimentario) derecho de un espécimen hembra de Mazama gouazoubira, revelando las medidas según von den Driesch (1976).
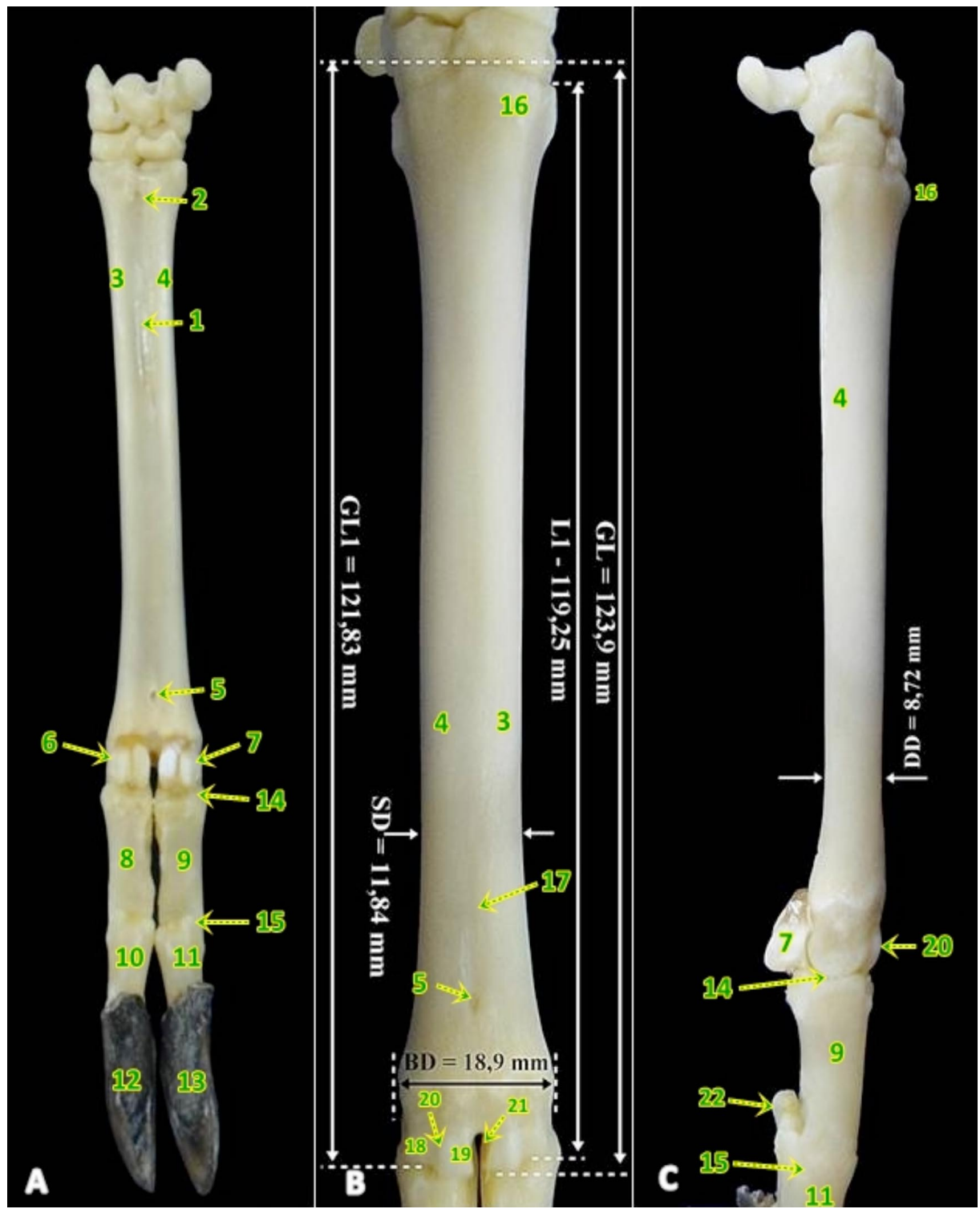

Fig. 6. Fotografía del esqueleto de la mano derecha de un espécimen hembra de Mazama gouazoubira (después de la eliminación de los metacarpianos y los dedos II y V) en vista palmar (A), dorsal (B) y lateral (C) muestra las medidas según von den Driesh (1976) y los accidentes anatómicos: surco longitudinal palmar (1), agujero metacarpiano proximal (2), metacarpiano III (3), metacarpiano IV (4), canal metacarpiano distal (5), sesamoideo proximal abaxial del dedo III (6) sesamoideo proximal abaxial del dedo IV (7), falange proximal del dedo III (8), falange proximal del dedo IV (9), falange media del dedo III (10), falange media del dedo IV (11), cara de la base de la pezuña del dedo III (12) y la cara de la base de la pezuña del dedo IV (13), articulación metacarpofalángica IV (14), articulación interfalángica proximal del IV dedo (15), tuberosidad metacarpiana (16), surco longitudinal dorsal (17), cóndilo abaxial del metacarpiano IV (18), cóndilo axial del metacarpiano IV (19), cresta sagital de la tróclea del metacarpiano IV (20) incisura intertroclear (21), tuberosidad flexora de la falange media (22). 

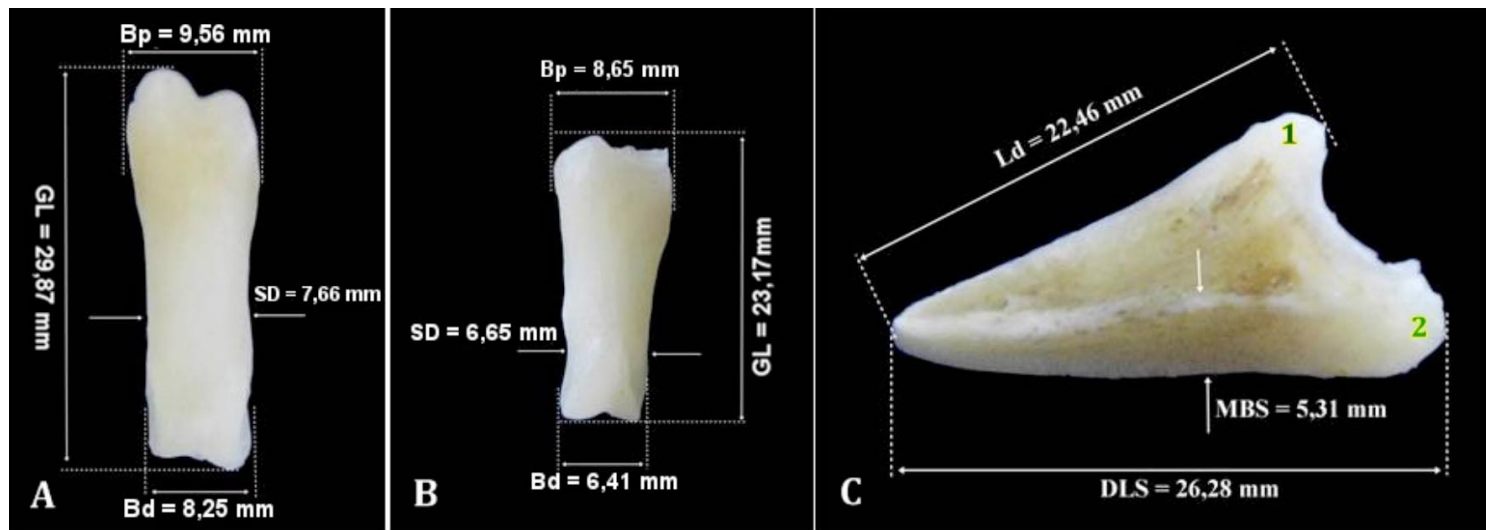

Fig. 7. Falanges de los dedos IV del miembro torácico derecho de un espécimen hembra de Mazama gouazoubira revelando las medidas de acuerdo con von den Driesh (1976) de la falange proximal en vista dorsal (A), falange media en vista dorsal (B) y falange distal en vista axial (C), con sus procesos extensores (1) y palmares (2).
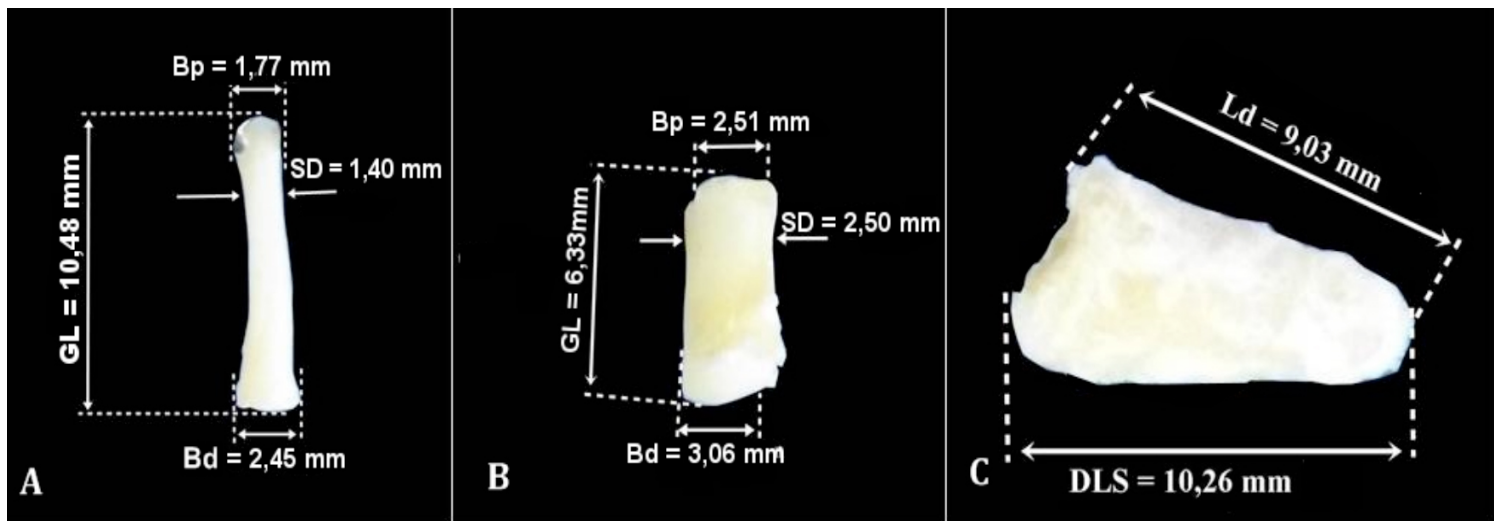

Fig. 8. Falanges del dedo II de un espécimen hembra de Mazama gouazoubira revelando las medidas según von den Driesh (1976) de la falange proximal en vista dorsal (A), falange media en vista dorsal (B) y la falange distal en vista abaxial (C).

Se observó la existencia de cuatro dedos (acropodio), dos principales (III y IV) (Fig. 7) que llegan hasta el suelo y dos rudimentarios (II y V) (Fig. 8). Todos los dedos tenían tres falanges, a diferencia de los rumiantes domésticos donde los dedos rudimentarios II y $\mathrm{V}$ tienen una o dos falanges (Nickel et al.). La formación de los cascos en todos los dedos concordó con lo descrito para M. gouazoubira por Rezende et al. Según Searfoss, la presencia de dos pequeñas pezuñas que tocan el suelo es característico de las especies que permanecen en terreno firme y seco, de planicies o zonas montañosas, lo que coincide con el tipo de terrenos habitados y la disposición del casco en Mazama gouazoubira. La disposición ósea del miembro torácico de M. gouazoubira es típica de mamíferos altamente cursoriales, en los cuales los dos dedos centrales están reforzados para recibir la fuerza del impacto con el suelo y los dígitos más periféricos se reducen o se pierden (Kardong, 2011). Del mismo modo, se puede obtener más firmeza con el mismo peso si el contenido esquelético se distribuye en un número menor de huesos. Al "clavar" los cascos de los miembros torácicos en el suelo, se facilita emplearlos como soporte para cambiar la dirección del tronco durante la locomoción saltatoria. Algunos de los mejores corredores y saltadores, y en particular, los más grandes, tienden a perder los dedos laterales y fusionar los elementos basales de los dedos, de origen compuesto. Por lo tanto, el metacarpiano de los cérvidos ungulados se reduce en respuesta al hábito de saltar. En su conjunto, el resultado es un miembro más ligero, delgado y resistente al impacto (Hildebrand \& Goslow Jr.; Kardong). Las imágenes obtenidas por radiología mostraron los aspectos anatomo-radiológicos de los huesos normales del miembro torácico de Mazama gouazoubira, y pueden servir como base para la interpretación de las pruebas de diagnóstico por imagen de los especímenes en cautiverio (Figs. 9, 10 y 11). 
Fig. 9. Radiografías de la escápula derecha en las proyecciones latero-medial (A) y cráneo-caudal (B), del húmero derecho en las proyecciones latero-medial (C) y cráneocaudal (D) y de la ulna y radio derechos en las proyecciones cráneo-caudal (E) y lateromedial (F) evidenciando la presentación radiográfica y los patrones de tensiones trabeculares normales para Mazama gouazoubira.
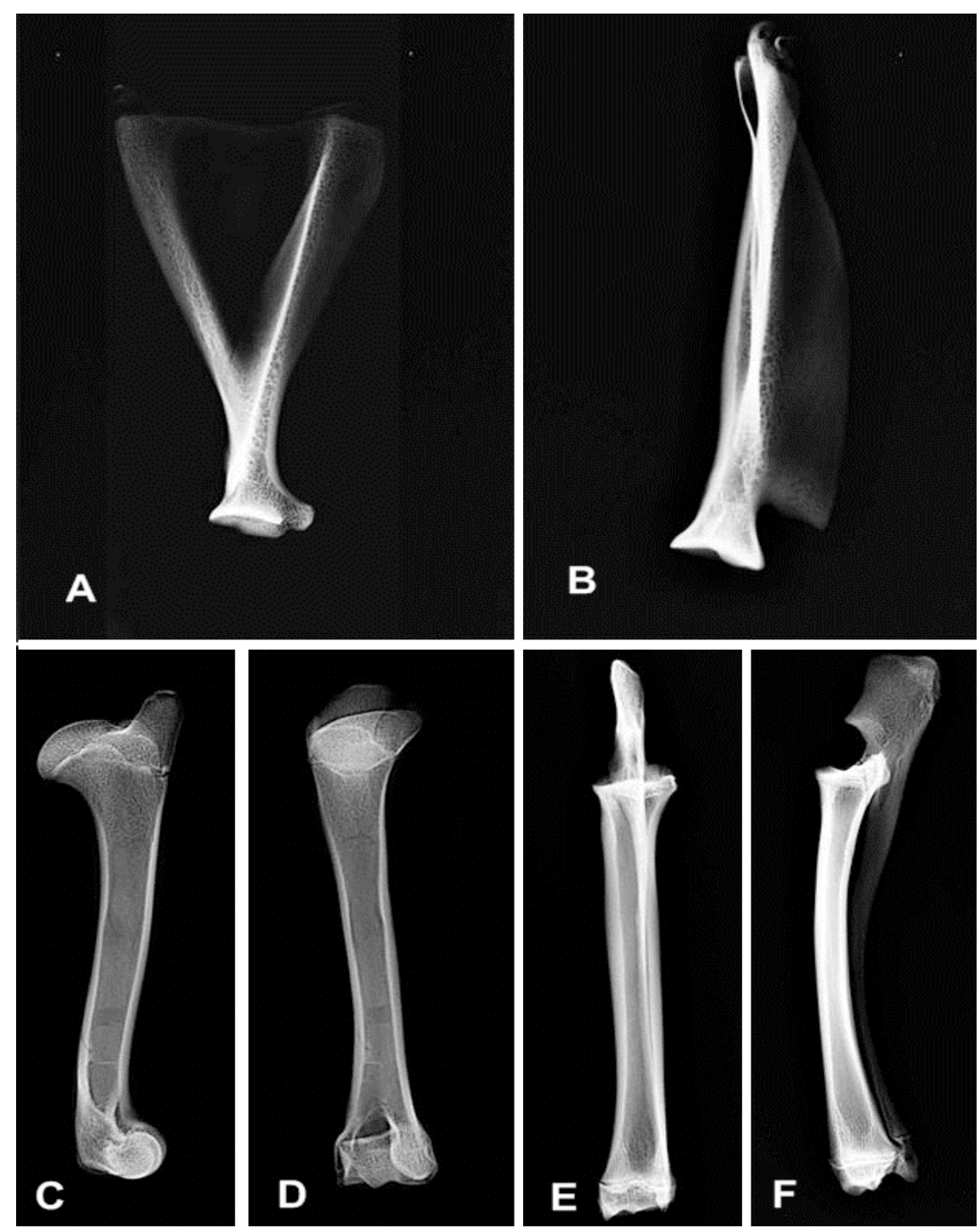
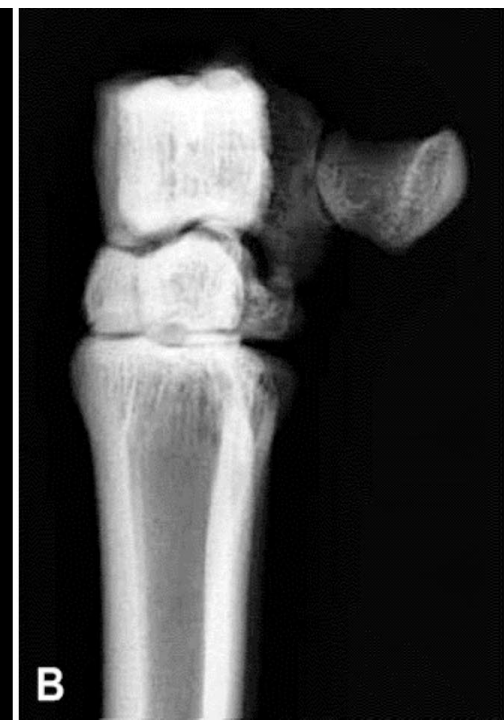

Fig. 10. Las radiografías de los huesos carpianos (antímero derecho) de un espécimen hembra de Mazama gouazoubira en vistas dorsopalmar (A), latero-medial. (B) y latero-medial en semiflexión (C) que muestran la manifestación radiográfica y los patrones de tensiones trabeculares normales. Las imágenes revelan el punto de unión entre el metacarpiano III y IV (flecha amarilla) y los espacios articulares radiocarpianos (flecha roja), intercarpiano (flecha azul) y carpometacarpiano (flecha verde). 

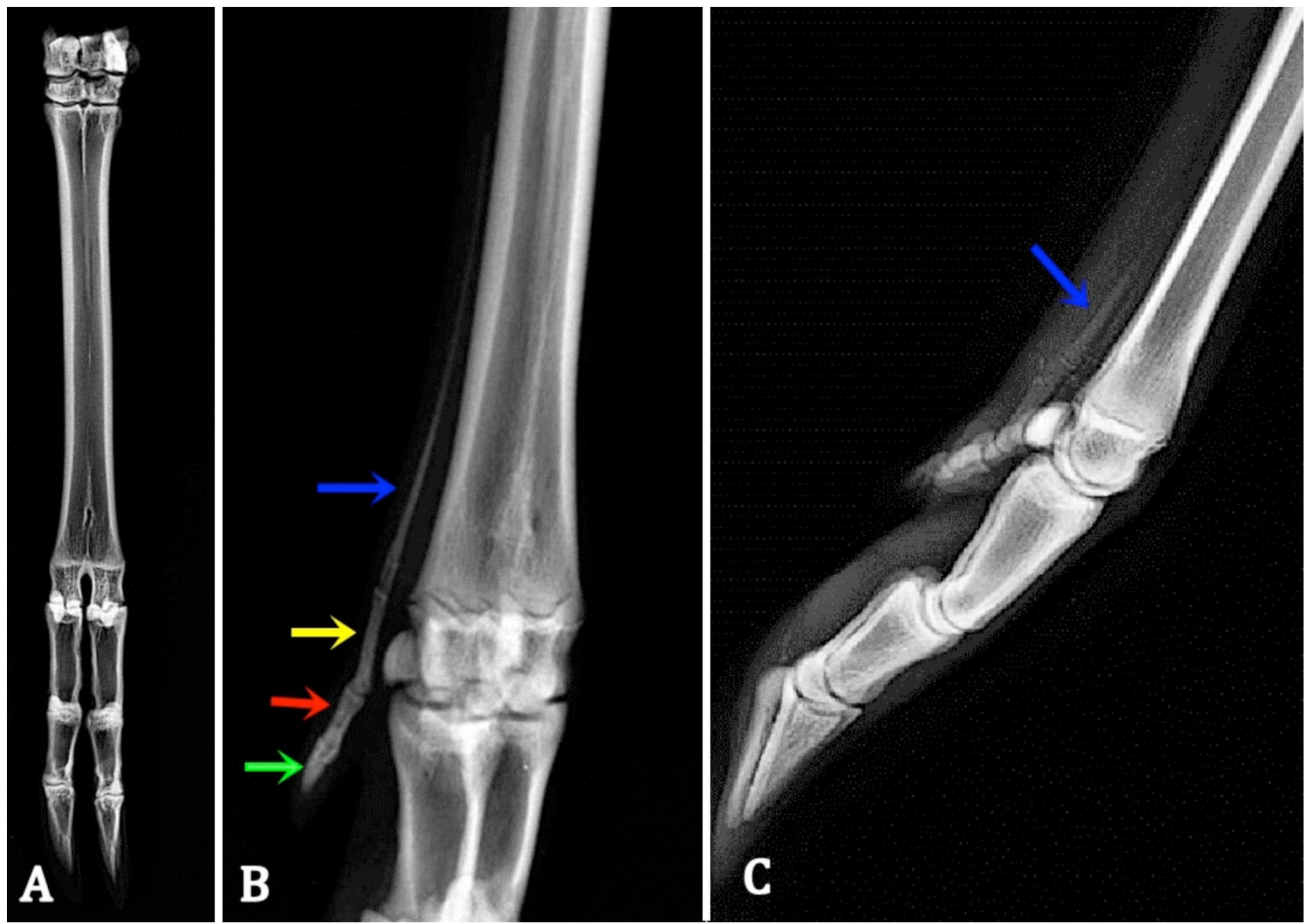

Fig. 11. Las radiografías del esqueleto de la mano de un espécimen hembra de Mazama gouazoubira en proyecciones dorsopalmar (A) después de la remoción de los dedos II y V, dorso-palmar oblicua (B) y latero-medial (C) mostrando el metacarpiano II (flecha azul), la falange proximal del dedo II (flecha amarilla), la falange media del dedo II (flecha roja) y la falange distal del dedo II (flecha verde).

Además, los patrones de tensiones trabeculares normales asociados al hueso esponjoso, concentrado en las epífisis y metáfisis de los huesos largos y en la región ventral de la escápula, se podrían visualizar claramente según lo recomendado por Emmerson (1986). Las radiografías permitieron revelar la ubicación y confirmar la fusión de las corticales axiales de los metacarpianos III y IV (Fig. 10A, flecha amarilla), las cuales eran casi imperceptibles a la inspección de la superficie del hueso.

No hubo relación estadísticamente significativa (nivel de a $=0,01$ ) en la osteometría entre antímeros, decidiéndose presentar el promedio de los valores obtenidos en los cuatro miembros analizados. Estas mediciones osteométricas tuvieron el propósito de especificar las dimensiones y pro- porciones de los huesos del miembro torácico de Mazama gouazoubira adulto. Por otra parte, se considera que estos datos pueden resultar útiles para que investigadores de otras áreas los comparen con muestras procedentes de otros biomas o regiones geográficas.

\section{AGRADECIMIENTOS}

Al Programa de Becas de Desarrollo Académico (PBDA) y de Educación Tutorial (PET) Veterinaria de la Universidad Federal de Pampa por las subvenciones concedidas al cuarto y quinto autor, respectivamente. 
SOUZA JUNIOR, P.; RIBEIRO, M. G. F.; DUTRA, L. J.; MATTOS, K.; CARVALHO, N. C.; ABIDU-FIGUEIREDO, M. \& SANTOS, A. L. Q. Osteology of the thoracic limb of South American Gray Brocket Mazama gouazoubira (Fischer, 1814) (Cetartiodactyla: Cervidae). Int. J. Morphol., $35(3): 1000-1009,2017$.

SUMMARY: This study aimed to describe anatomical features of the forelimb skeleton of Mazama gouazoubira in order to establish morphofunctional activity. Forelimbs of four young adult specimens were used to this end. The bones were prepared by boiling and cleared in a solution of hydrogen peroxide for comparative descriptions, osteometrics measurements and digital radiographs. Compared to domestic ruminants, the scapula proved to be wide and flat, with a small acromion, reduced coracoid process and lacking of spine tuberosity. The humerus had an elongated shaft with a rounded cross-section and discrete non-articular projections. The ulna accompanied the radio to the distal epiphysis and formed a long and narrow, proximally placed interosseous space without a vascular groove. The skeleton of the hand presented the rudimentary metacarpals II and V and the welldeveloped III and IV fused metacarpals, which is a typical telemetacarpal arrangement of close phylogenetically deer species from the New World. There were four fingers, each one with three phalanges; two main (III and IV) touching the ground and two rudimentary (II and V). Radiographs allowed visualizing patterns of normal trabecular tensions and osteometrics enabled to establish ratios for comparative purposes. Adaptations of skeletal forelimb to favor cursorial saltatory locomotion were typically identified.

KEY WORDS: Animal anatomy; Cervids; Osteometrics; Telemetacarpals; Wild animals.

\section{REFERENCIAS BIBLIOGRÁFICAS}

Black-Decima, P. A. \& Vogliotti, A. Mazama gouazoubira. The IUCN Red List of Threatened Species 2016:e.T29620A22154584, 2016.

Borelli, V. \& Gonçalves, A. M. M. Aspectos morfológicos dos componentes do funículo espermático do veado-mateiro (Mazama americana). J. Health Sci. Inst., 29(4):294-8, 2011.

Borges, E. M.; Machado, M. R. F.; de Oliveira, F. S.; de Souza, W. M. \& Duarte, J. M. B. Aspectos morfológicos do fígado do cervo-do-pantanal (Blastocerus dichotomus). Braz. J. Vet. Res. Anim. Sci., 39(2):78-80, 2002.

Brooke, V. On the classification of the Cervidœ, with a synopsis of the existing species. Proc. Zool. Soc. Lond., 46(1):883-928, 1878.

Cap, H.; Aulagnier, S. \& Deleporte, P. The phylogeny and behavior of Cervidae (Ruminantia Pecora). Ethol. Ecol. Evol., 14(3):199-216, 2002.

Cavagna, G. A.; Heglund, N. C. \& Taylor, C. R. Mechanical work in terrestrial locomotion: two basic mechanisms for minimizing energy expenditure. Am. J. Physiol., 233(5):R243-61, 1977.

Costa, K. L. C. Avaliação morfofuncional do testículo de veado catingueiro (Mazama gouazoubira Fisher, 1814). Dissertação (Mestrado em Biologia Animal). Viçosa, Universidade Federal de Viçosa, 2009.

Emmerson, M. A. Anatomia na Radiologia. In: Getty, R. (Ed.). Anatomia dos Animais Domésticos. Vol. 1. $5^{\text {th }}$ ed. Rio de Janeiro, Guanabara Koogan, 1986. pp.5-14.

Ferreira, A. O.; Morini, A. C.; Favaron, P. O.; Passos, C. C.; Campos, D. B.; Miglino, M. A. \& Guerra, R. R. Avaliação morfológica das membranas fetais e da placenta de Mazama gouazoubira (veado-catingueiro) de vida livre no terço inicial da gestação. Pesq. Vet. Bras., 31(7):631-5, 2011.

Geist, V. Deer of the World: Their Evolution, Behavior and Ecology. Mechanicsburg, Stackpole Books, 1998.

Gilbert, C.; Ropiquet, A. \& Hassanin, A. Mitochondrial and nuclear phylogenies of Cervidae (Mammalia, Ruminantia): Systematics, morphology, and biogeography. Mol. Phylogenet. Evol., 40(1):101-17, 2006.

Guimarães, G. C.; Rosa, M. C. B.; Lopes, G. C.; Narciso, T. P. \& Oliveira, F. S. Formação da veia jugular externa no veado catingueiro (Mazama gouazoubira). Biotemas, 25(4):289-92, 2012.
Hildebrand, M. \& Goslow Jr., G. E. Análise da Estrutura dos Vertebrados. $2^{\text {nd }}$ ed. São Paulo, Atheneu, 2006.

International Commitee on Veterinary Gross Anatomical Nomenclature (ICVGAN). Nomina Anatomica Veterinaria. $5^{\text {th }}$ ed. Hannover, Editorial Commitee, 2012.

Kardong, K. V. Vertebrados: Anatomia Comparada, Função e Evolução. $5^{\text {th }}$ ed. São Paulo, Roca, 2011.

Kokubun, H. S.; Esper, G. V. Z.; Franciolli, A. L. R.; Silva, F. M. O.; Rici, R. E. G. \& Miglino, M. A. Estudo histológico e comparativo das papilas linguais dos cervídeos Mazama americana e Mazama gouazoubira por microscopia de luz e eletrônica de varredura. Pesqui. Vet. Bras., 32(10):1061-6, 2002.

Liebich, H. G.; Maierl, J. \& König, H. E. Membros Torácicos ou Anteriores (Membra Thoracica). In: König, H. E. \& Liebich, H. G. (Eds.). Anatomia dos Animais Domésticos. $4^{\text {th }}$ ed. Porto Alegre, Artmed, 2011. pp.165-234.

Liem, K. F.; Bemis, W. E.; Walker Jr., W. F. \& Grande, L. Anatomia Funcional dos Vertebrados: Uma Perspectiva Evolutiva. $3^{\text {rd }}$ ed. São Paulo, Cengage, 2013.

Lima, F. C.; Santos, A. L. Q.; Lima, B. C.; Vieira, L. G. \& Hirano, L. Q. L. Topographic anatomy of the spinal cord and vertebromedullary relationships in Mazama gouazoubira Fisher, 1814 (Artiodactyla; Cervidae). Acta Sci. Biol. Sci., 32(2):189-94, 2010.

Mähler Junior, J. F. K. \& Schneider, M. Ungulados. In: Bencke, G. A.; Fontana, C. S.; Reis, R. E. (Eds.). Livro Vermelho da Fauna Ameaçada de Extinção no Rio Grande do Sul. Porto Alegre, EDIPUCRS, 2003.

Martins, T. M. M.; Pereira, K. F.; Lima, F. C.; Santos, A. L. Q. \& Malysz, T. Origem e distribuição do nervo isquiático no veado-catingueiro (Mazama gouazoubira). Pesq. Vet. Bras., 33(2):273-8, 2013.

Nickel, R.; Schummer, A.; Seiferle, E.; Wilkens, H.; Wille, K. H. \& Frewein, J. The Anatomy of the Domestic Animals: The Locomotor System of the Domestic Mammals. Berlin, Verlag Paul Parey, 1986.

Rezende, L. C.; dos Santos, J. P. A.; Urbani, L. T.; Galdos-Riveros, A. C. \& Miglino, M. A. Análise morfológica do aparelho ungueal do veadocatingueiro (Mazama gouazoubira, Fischer, 1814) (Artiodactyla, Cervidae). Ciênc. Anim. Bras., 14(2):230-6, 2013.

Searfoss, G. Skulls and Bones. Mechanicsburg, Stackpole Books, 1995.

Sisson, S. Osteologia Ruminante. In: Getty, R. (Ed.). Anatomia dos Animais Domésticos. Vol. 1. $5^{\text {th }}$ ed., Rio de Janeiro, Guanabara Koogan, 1986. pp.693-735.

Teixeira, M. B.; Lepkoski, E.; Vieira, R.; Fernandes, G.; Dornelles, R.; Ávila, M.; Koenemann, J. \& Oliveira, E. Ocorrência de Mazama gouazoubira (Fischer, 1814) na região sudoeste do Rio Grande do Sul, pampa brasileiro. Rev. Bras. Zoociências, 12(2):181-6, 2010.

Tiepolo, L. M. \& Tomas, W. M. Ordem Artiodactyla. In: Reis, N. R.; Lima, I. P; Pedro, W. A. \& Perachi, A. L. (Eds.). Mamíferos do Brasil. Londrina, Nélio R. dos Reis, 2006. pp.283-304.

Varela, G. Osteología y Miología de lós Miembros Anterior y Posterior del Venado de Campo (Ozotoceros Bezoarticus). Dissertação (Licenciatura em Ciências Biológicas). Montevideo, Universidad de la Republica, 2010.

Vieira, L. G.; Ribeiro, P. R. Q.; Lima, M. O.; de Souza, R. R.; Valdes, S. A. C. \& Santos, A. L. Q. Origens e ramificações do plexo braquial do veado catingueiro Mazama gouazoubira (Artiodactyla: Cervidae). Biotemas, 26(1):137-46, 2013.

von den Driesch, A. A Guide to the Measurement of Animal Bones from Archaeological Sites. Massachussets, Peabody Museum Bulletins, 1976.

\section{Dirección para correspondencia}

Prof. Paulo de Souza Junior

Laboratório de Anatomia Animal da UNIPAMPA

Rodovia BR 472, km 592,

Caixa Postal 118,

CEP 97508-000

Uruguaiana, RS, BRAZIL

Recibido : 01-02-2017

Aceptado: 07-04-2017

E-mail: paulosouza@unipampa.edu.br 